\title{
Cannabispolitik: und sie dreht sich doch!
}

\author{
Daniel Beutler-Hohenberger ${ }^{\text {, }}$ \\ Jean Henri Dunant ${ }^{b}$, \\ Francis Thévot ${ }^{c}$, Urs Jundt ${ }^{d}$ \\ a Dr. med., Co-Präsident DaD, \\ Mühlethurnen \\ b PD Dr. med., Nationalrat, Basel \\ c Dr. med., Député Grand Conseil \\ VD, Maracon \\ d Dr. med., Arbon
}

Der Artikel der Kollegen Hämmig, Savary und Theunert (im folgenden als Autoren bezeichnet) spiegelt eine liberale Grundhaltung in Sachen Cannabispolitik, die entsprechende Fragen aufwirft [1]. Wie nüchtern und ideologiefrei ist die Haltung der Autoren? Wie wissenschaftlich und evidenzbasiert ist ihre Sichtweise? Zugegeben, über Cannabis kann man geteilter Meinung sein, je nachdem wessen Konsum man betrachtet. Der medizinische Cannabisgebrauch von MS-Kranken entspricht nicht demjenigen vulnerabler, psychosegefährdeter Jugendlicher, und der Gelegenheitskonsum der Alt-68er lässt sich kaum mit dem exzessiven Indoor-Konsum des jungen Lehrlings mit Schulschwierigkeiten vergleichen. Aber sobald man sich mit den Hanfaktivisten solidarisiert, ist man nicht mehr ideologiefrei! Die Hanfinitiative steht für ein Gedankengut, das der Philosophie von «Sex, Drugs and Rock'n'roll» entspringt und so eine gesellschaftlich destruktive Ideologie propagiert, die weit über liberales Gedankengut hinausgeht [2]. Das Mitmarschieren einiger bürgerlicher Exponenten ändert nichts an dieser Tatsache. Auch der

\section{Die Hanfinitiative steht für ein Gedankengut, das der Philosophie von «Sex, Drugs and Rock'n'Roll» entspringt}

Korrespondenz:

Dr. med. Daniel Beutler-Hohenberger Bahnhofstrasse 50

3127 Mühlethurnen

dan.beutler@hin.ch manipulative Untertitel «wirksamer Jugendschutz» ist blosse Augenwischerei. Der einzige wirksame Jugendschutz wäre die Solidarität der Hanffreunde mit den gefährdeten jugendlichen Konsumenten, nämlich der Verzicht auf den straffreien Cannabiskonsum [3].

Die empirische Datenlage verdichtet sich eindeutig in die Richtung, dass der Cannabiskonsum weitaus schädlicher ist, als bisher angenommen wurde! Es gibt sehr wohl neuere wissenschaftliche Erkenntnisse, die ein Beibehalten des Cannabisverbots, d.h. das Umsetzen der geltenden Rechtssprechung, rechtfertigen. Das zitierte Expertenrating ist nutzlos und bringt keine neuen Erkenntnisse. Die zugrundeliegende WHO-Klassifikation wird der Cannabisproblematik nicht gerecht, weil der ausgeprägten interindividuellen Unterschiedlichkeit der Wirkung von Tetrahydrocannabinol (THC) auf Körper und Psyche zu wenig Beachtung geschenkt wird. Cannabis ist eine Droge, die sich nicht nur auf die Konsumenten, sondern immer mehr auch auf die Gesellschaft negativ auswirkt [4].

Die Datenlage zur Wirkung der Repression ist uneinheitlich, wobei beachtet werden muss, dass die gesetzlichen Vorgaben in keiner Weise der effektiven Rechtspraxis entsprechen müssen. Besonders die schweizerische Drogenpolitik ist ein Musterfall einer nicht umgesetzten Gesetzgebung. In Italien beispielsweise schien die Entkriminalisierung 1975 den Anstieg des Drogenkonsums zu begünstigen [5]. In den USA hatte die Liberalisierung der Rechtspraxis in einigen Staaten keinen Einfluss auf das Konsumverhalten, so dass die Gesetze wieder verschärft wurden [6]. Besonders erwähnenswert ist, dass sich die Niederlande als Vorreiterin einer liberalen Drogenpolitik wieder vermehrt der Repression zuwendet [7]. In Grossbritannien wurde Cannabis sogar von einer Droge der Kategorie C in die höhere Kategorie B reklassifiziert, was mit schärferen Restriktionen und höheren Bussen verbunden ist [8].

Die Tatsache, dass im Kapitel «Atemwege» (S. 749) am Schluss erwähnt wird, Cannabis könne antikarzinomatöse Aktivität entwickeln, hingegen verschwiegen wird, dass neuere Arbeiten eindeutig das karzinogene Potential der inhalierten Cannabinoide (unabhängig vom Tabak) belegen $[9,10]$, ist zumindest unwissenschaftlich. Auch im Abschnitt «Kognitive Entwicklung» (S. 749) werden wichtige neue Erkenntnisse, insbesondere aufgrund neurophysiologischer Grundlagenarbeiten, verschwiegen [11, 12]. Cannabis hinterlässt besonders in der Schwangerschaft schon nach moderatem Konsum irreversible Veränderungen in den fötalen Hirnen, die sich nach neuesten Erkenntnissen vor allem auf die emotionale Entwicklung dieser Kinder auswirken. Solche Fakten einfach auszublenden ist unseriös und widerspricht dem Anspruch einer evidenzbasierten Drogenmedizin.

Die Aussagen im Abschnitt «Cannabis und Schizophrenien» (S. 750), Cannabis könne eventuell den Krankheitsausbruch beschleunigen und den Krankheitsverlauf negativ beeinträchtigen und es bestehe keine Evidenz für die These einer kausalen Beziehung zwischen Cannabiskonsum und Auslösung von Schizophrenien, 
sind unzutreffend und widersprechen eindeutig der aktuellen Datenlage. Spätestens seit der «Swedish conscript cohort study» von Zammit et al. ( $\mathrm{n}=15000$ !) [13] und «Dutch NEMESIS Sample» von van Os et al. $(\mathrm{n}=4000)$ [14], die ein mindestens drei- bis sechsfach erhöhtes Risiko für Cannabiskonsumenten, an einer Schizophrenie zu erkranken, ohne jeden Zweifel belegten, dienten alle weiteren Studien nur noch der Bestätigung dieser These. Die lange vermisste epidemiologische Bestätigung wurde durch die «Zürcher Studie» von Rössler et al. zweifelsfrei erbracht, zumal sie durch ein ausgeklügeltes statistisches Verfahren alle anderen soziokulturellen
Schizophrenie besteht, was unter anderem hinsichtlich Suizidproblematik eine neue gesundheitspolitische Herausforderung bedeutet. Die Behauptung, es sei noch nie jemand am Cannabiskonsum verstorben, ist falsch!

Die bestehende Rechtsunsicherheit muss beseitigt und die Glaubwürdigkeit der Behörden wiederhergestellt werden - das ist unbestritten. Die Hanfinitiative mit ihrem fatalen Signal an die Jugend ist zweifelsohne der falsche Weg. Namentlich zum Jugendschutz wurde seitens der Hanfbefürworter im Verlauf der bisherigen politischen Diskussion kein einziger praktikabler Weg zur Umsetzung aufgezeigt. Das dürfte auch

\section{Cannabis ist eine Droge, die sich nicht nur auf die Konsumenten, sondern immer mehr auch auf die Gesellschaft negativ auswirkt}

Einflüsse geschickt ausblendete [15]. Weiter wird eine australische Untersuchung zitiert, die wiederum die vermeintlich fehlenden epidemiologischen Auswirkungen des Cannabiskonsums anprangert [16]. Hier ist zu bemerken, dass Klinikeinweisungen nur bedingt ein Mass für die Prävalenz der Schizophrenie darstellen. Einerseits bilden die ambulant behandelten psychotischen Störungen sicher eine erhebliche Dunkelziffer, zumal die modernen Neuroleptika eine ausserklinische Behandlung eher ermöglichen. Andererseits führen sub- oder präpsychotische Zustände, wie sie bei Cannabiskonsumenten vorkommen können, oft nicht zu einer Klinikeinweisung, so dass diese statistisch nicht erfasst werden. Die akuten, klinikbedürftigen Schizophrenien bilden also höchstens die Spitze des Eisbergs. In der «Soteria», einer Institution der «Interessengemeinschaft Sozialpsychiatrie» in Bern, stellte man in den letzten Jahren eine überproportionale Zunahme von jungen Cannabiskonsumenten unter den an Schizophrenie Erkrankten fest, so dass sogar ein spezifisches, abstinenzorientiertes Behandlungskonzept eingeführt wurde [17]. Für dieses neue Konzept erhielt die Institution von der Schweizerischen Gesellschaft für Psychiatrie und Psychotherapie den «Prix Perspective».

Die Verharmlosung von Cannabis und die permissive Handhabung des Betäubungsmittelgesetzes haben $\mathrm{zu}$ einer beängstigenden $\mathrm{Zu}$ nahme des Drogenkonsums in der Schweiz beigetragen. Bezüglich gesundheitlicher Folgeschäden verdichtet sich die wissenschaftliche Datenlage eindeutig dahingehend, dass ein $\mathrm{Zu}$ sammenhang zwischen Cannabiskonsum und schwierig sein, denn die Beschaffungswege der unter 18jährigen Cannabiskonsumenten sind gesetzlich kaum fassbar, geschweige denn regulierbar [18].

Was die Autoren in ihrem Schlussvotum als fachliche Perspektive bezeichnen, entspricht de facto einer selektiven, wenn nicht sogar tendenziösen Wahrnehmung. Die Interpretation der wissenschaftlichen Evidenz weist eine entsprechende Unschärfe auf, die der Korrektur bedarf. Die abstinenzorientierten Organisationen und Drogenfachleute der Schweiz fordern eine glaubwürdige Drogenpolitik, frei von Ideologien, aber werteorientiert, nicht nach Augenmass, sondern evidenzbasiert - Hände weg von Drogen!

\section{Literatur}

1 Hämmig R, Savary JF, Theunert M. Cannabispolitik: weder dämonisieren noch banalisieren. Schweiz Ärztezeitung. 2008;89(17):748-52.

2 Regli D. Die 68er-Falle - Fluchtwege aus dem Desaster der Neuen Linken. Zürich: Artesio; 2005.

3 Beutler D. Forum «Der Bund», 16. August 2007.

4 Facts. Bekifft mit 12 - eine Tüte zum Frühstück. 2006;(1):24-31.

5 Solivetti LM. Drug Use Criminalization vs Decriminalization: An Analysis in the Light of the Italian Experience. BAG-Report, Rom 2001. *

6 Single E, Christie P. The Consequences of Cannabis Decriminalization in Australia an the U.S. BAGReport, Bern 2001.*

7 «Zahl der Coffeeshops sinkt». Spiegel Online. 22. Dezember 2004.

8 United Kingdom Reclassifies Marijuana. The Times, 9. Mai 2008. 
9 Aldington S, Harwood M, Cox B, Weatherall M, Beckert L, Hansell A, et al. Cannabis use and risk of lung cancer: a case-control study. Eur Respir J. 2008;31:280-6.

10 Brambilla C, Colonna M. Cannabis: the next villain on the lung cancer battlefield? Eur Respir J. 2008; $31: 227-8$

11 Laviolette SR, Grace AA. Cannabinoids potentiate emotional learning plasticity in neurons of the medial prefrontal cortex through basolateral amygdala inputs. J Neurosci. 2006;26(24):6458-68.

12 Berghuis P, Harkany T, et al. Hardwiring the brain: endocannabinoids shape neuronal connectivity. Science. 2007;316(5828):1212-6.

13 Zammit S, Allebeck P, Andreasson S, Lunberg I, Lewis G. Self reported cannabis use as a risk factor for schizophrenia in Swedish conscripts of 1969: historical cohort study. Br Med J. 2002;325:1199.
14 van Os J, Bak M, Hanssen M, Bijl LV, de Graaf R, Verdoux H. Cannabis use and psychosis: a longitudinal population-based study. Am J Epidemiol. 2002;156:319-27.

15 Ajdacic-Gross V, Lauber C, Warnke I, Haker H, Murray RM, Rössler W. Changing incidence of psychotic disorders among the young in Zurich. Schizophrenia Res. 2007;95:9-18.

16 Degenhardt L, Hall W, Lynskey M. Testing the hypotheses about the relationship between cannabis use and psychosis. Drug Alcohol Depend. 2003;71(1):37-48.

17 Mitteilung der Interessengemeinschaft Sozialpsychiatrie in Bern, April 2007.

18 Müller et al. Cannabis auf der Schwelle zum legalen Rauschmittel. Schweizerische Fachstelle für Alkohol- und andere Drogenprobleme - SFA. Publikation 2001.

\section{Replik}

\section{Die Dres. Beutler-Hohenberger, Dunant, Thévot und Jundt liefern mit ihrer Replik ein Muster kreativer Argumentationsführung, die einer fachlichen und einer politischen Antwort bedarf.}

\section{Robert Hämmiga , Jean-Félix Savary ${ }^{b}$,} Markus Theunert ${ }^{c}$

a Präsident der Schweizerischen Gesellschaft für Suchtmedizin (www.ssam.ch)

b Generalsekretär Groupement Romand d'Etudes des Addictions (www.grea.ch)

c Generalsekretär Fachverband Sucht (www.fachverbandsucht.ch)

Korrespondenz:

Dr. med. Robert Hämmig

Universitäre Psychiatrische Dienste

Bern

Funktionsbereich Sucht

Ziegelstrasse 7

CH-3010 Bern

haemmig@spk.unibe.ch

\section{Die fachliche Perspektive}

Die Autoren weisen richtigerweise darauf hin, dass auch in einer «evidence-based medicine» die publizierten Befunde bewertet werden müssen. Die von den Autoren angeführte Evidenz für die besondere Gefährlichkeit von Cannabis und die Belege dafür, dass Cannabis Schizophrenien erzeugt, haben alle den Makel, dass sie komplexe Lebensrealitäten heutiger gesellschaftlicher Bedingungen auf einen einzigen Faktor reduzieren und die gefundenen Korrelationen im Sinne von «post hoc - propter hoc» als Kausalität präsentieren. Ein einfacher Gedankengang zeigt, wie Korrelationen zu Kausalitäten zurechtgebogen werden: Die Schizophrenie-Inzidenz ist in den letzten 30 Jahren mit gewissen Schwankungen stabil bis leicht rückläufig. In dieser Zeit hat sich der Cannabiskonsum etwa verfünffacht. Würde Cannabis Schizophrenien auslösen, die ohne Cannabiskonsum hätten verhindert (und nicht bloss verzögert) werden können, so müsste die Schizophrenie-Inzidenz zwingend parallel zum Cannabiskonsum gestiegen sein.

Niemand behauptet, dass exzessiver Cannabiskonsum unproblematisch sei. Dies kann heute sogar als Binsenwahrheit angesehen wer- den und wurde wissenschaftlich schon im «Indian Hemp Commission Report» von 1894 belegt. Zudem wurden in den letzten Jahren auf den Drogenberatungsstellen der Schweiz vermehrt junge exzessive Cannabiskonsumenten vorstellig, die in ihrer Gesamtproblematik den jungen Heroinkonsumenten der 70er und 80er Jahre ähneln, bei gleichzeitigem Rückgang der letzteren. Als neuste Entwicklung lässt sich feststellen, dass die Zahl der Cannabiskonsumenten zurückgeht, bei gleichzeitiger Zunahme der jugendlichen «Kampftrinker» und «Komasäufer». Diese Beobachtungen weisen darauf hin, dass Gesellschaften dynamische Gebilde sind und dass es letztlich nicht primär um Substanzen geht, sondern darum, die zugrundeliegenden Ursachen anzugehen.

\section{Die politische Perspektive}

Die Autoren propagieren als «ideologiefreie und werteorientierte» Lösung eine Verstärkung der Prohibition. Dazu lässt sich folgendes sagen:

- Die «Ideologiefreiheit» der Autoren darf ruhig angezweifelt werden: Ein kurzer Blick auf die Website des «Dachverbandes abstinenzorientierter Drogenpolitik», in dessen Na- 
men die Replik verfasst wurde, zeigt auf, dass es sich bei seinen Exponenten genau um die Leute handelt, die sich in den letzten Jahren immer gegen die offizielle Drogenpolitik und alle Weiterentwicklungen gestellt haben («Zürcher Flügel» der SVP, EDU, FPS, Schweizer Ärzte gegen Drogen, Jugend ohne Drogen, Eltern gegen Drogen, Aids-Aufklärung Schweiz usw.). So versuchen diese Leute derzeit auch mit einem Referendum die mühsam im Parlament austarierte Betäubungsmittelgesetzesrevision $\mathrm{zu}$ torpedieren. Ihr Ziel ist gemäss Leitbild, den «Gebrauch von Rauschgiften (Drogen) zu ächten». Das mag man unterstützen oder nicht, ideologiefrei und «evidence-based» ist ein solches Ansinnen ganz bestimmt nicht.

- Die heutige Drogenproblematik in allen Ländern der Welt, also auch in der Schweiz, hat sich unter der weltweiten Politik der Drogenprohibition entwickelt. Die Drogen in ihrer Gesamtheit (inkl. Alkohol, Tabak, betäubende und stimulierende pharmazeutische Produkte) sind trotz Verboten im Vormarsch. Wie die Geschichte aufzeigt, ist es bis jetzt nur in totalitären Regimes wie im Kommunismus maoistischer Prägung gelungen, Drogen in wesentlichem Ausmass einzudämmen. Dies kann sicher kein Gesellschaftsmodell sein, das wir uns wünschen. Zudem hat die Lockerung der persönlichen Einschränkungen auch in China in kürzester Zeit zu einer massiven Zunahme der Drogenprobleme geführt.

- Eine Lösung, die nicht zum gewünschten Erfolg führt, führt auch mit «mehr des Selben» nicht zum gewünschten Ziel.

- Prohibition ist teuer. Drogenabteilungen hat es vor 1970 weder bei der Polizei noch bei der Justiz gegeben, heute gelten sie in der Schweiz als eine undiskutierte Selbstverständlichkeit. Erstaunlicherweise gibt es kaum Kosten-Nutzen-Analysen zu dieser Thematik. Die RAND Corporation, ein mit der US Air Force verbundener «Think Tank», hat 1994 in bezug auf den Kokainkonsum in den USA errechnet, dass für einen Dollar eingesetztes Steuergeld wesentlich mehr Kokainkonsum verhindert werden kann, wenn dieser Dollar statt in die Strafverfolgung in die Therapie investiert wird [1].

- Strafrecht und entsprechend die Strafandrohungen im Betäubungsmittelrecht sind darauf ausgelegt, den Leuten Schaden zuzufügen (nach dem Motto «Durch Schaden wird man klug»). Aus Sicht der ärztlichen Ethik ist deshalb dieser Vorgehensweise mit der gebührenden Vorsicht $\mathrm{zu}$ begegnen (Prinzip des «noli nocere»). Betrifft die Sanktion Verhalten, die geeignet sind, andere zu schädigen («public vice» wie Drogenhandel, Fahren unter Alkoholeinfluss usw.), ist dies und die damit verbundene gesellschaftliche Marginalisierung durchaus ethisch zu rechtfertigen. Anders verhält es sich bei Leuten, die sich schon ohnehin durch Drogenkonsum schädigen («private vice»). Ziel müsste es hier sein, sie nicht noch zusätzlich mit strafrechtlichen Massnahmen zu schädigen und zu marginalisieren, sondern ihnen $\mathrm{zu}$ helfen, sich wieder in die gesellschaftlichen Rahmenbedingungen zu integrieren. Zudem sind die durchs Strafrecht erzielten Schädigungen nicht zielgenau: Sie treffen auch das Umfeld der Betroffenen.

Wer jetzt diesen Ausführungen ein Plädoyer für den freien und ungehinderten Drogenmarkt entnimmt, liegt falsch. Neoliberale ökonomische Modelle, wie sie z.B. von Milton Friedman propagiert werden, sind genauso keine Lösung wie die Prohibition. Es braucht staatliche Regulierungen, und dass diese auch ohne Prohibition erfolgreich sein können, zeigt die «Branntweinpest» des 19. Jahrhunderts und die getroffenen Massnahmen, die zu einer anhaltenden Konsumreduktion geführt haben [2]. Dieses historische Beispiel zeigt auch auf, dass es keine einfachen Lösungen gibt und dass Lösungen in einem langwierigen und mühsamen politischen Prozess gefunden werden müssen. Diese Erkenntnis hat sich heute auch bei der WHO durchgesetzt [3].

Die Cannabisprohibition verhindert diesen Prozess und die Hanfinitiative würde bei ihrer Annahme die Rahmenbedingungen schaffen, die eine solche Lösungsfindung überhaupt erst ermöglichen.

\section{Literatur}

1 Rydell CP, Everingham SS. Controlling Cocaine: Supply Versus Demand Programs. RAND; 1994.

2 Merki CM. Zur neueren Geschichte der psychoaktiven Substanzen. Bericht zuhanden der Eidgenössischen Kommission für Drogenfragen (EKDF). Historisches Institut der Universität Bern; 2002.

3 Wilkinson R, Marmot M. Soziale Determinanten von Gesundheit: die Fakten. Zweite Ausgabe. Kopenhagen: WHO-Regionalbüro für Europa; 2004 . 